\title{
sciendo
}

\section{The Yo-Yo Intermittent Endurance Level 2 Test: Reliability of Performance Scores, Physiological Responses and Overload Characteristics in Competitive Soccer, Basketball and Volleyball Players}

\author{
by \\ Konstantinos Papanikolaou ${ }^{1}$, Athanasios Chatzinikolaou², Theodoros Pontidis², \\ Alexandra Avloniti², Chariklia K. Deli ${ }^{1}$, Diamanda Leontsini², Dimitrios \\ Draganidis ${ }^{1}$, Panagiotis D. Tsimeas ${ }^{1}$, Lefteris Rafailakis ${ }^{1}$, Athanasios Z. Jamurtas ${ }^{1}$, \\ Peter Krustrup ${ }^{3,4}$, Magni Mohr5,6, Ioannis G. Fatouros ${ }^{1}$
}

The purpose of this study was to evaluate the reliability and reproducibility of the physiological and overload features of the Yo-Yo intermittent endurance test level 2 (Yo-Yo IE2) in competitive male soccer $(n=20)$, basketball $(n=$ 11), and volleyball players $(n=10)$. The participants completed Yo-Yo IE2 tests on three separate occasions with assessment of performance, heart rate, running speed, accelerations, decelerations and body load using GPS instrumentation. The intra-class correlation coefficient index, confidence intervals and coefficients of variation were calculated to assess the reliability of the test. Intra-class correlation coefficients for test-retest trials in the total sample ranged from large to nearly perfect (total distance: 0.896; mean speed: 0.535; maximum speed: 0.715; mean HR: 0.876; maximum HR: 0.866; body load: 0.865). The coefficients of variation for distance, mean speed, HR response, as well as acceleration and deceleration scores for test-retest trials ranged from 1.2 to $12.5 \%$ with no differences observed among particular sport disciplines. The CV for shuttles performed ranged from 4.4 to $5.5 \%$ in all sports. Similar results were obtained for the three different categories of players tested. These results suggest that the Yo-Yo IE2 test appears to be a reliable alternative for evaluating the ability to perform intermittent high-intensity running in different outdoor and indoor team sports. Players may need one or two familiarization tests to ensure valid assessment of intermittent endurance capacity. It appears that the Yo-Yo IE2 test incorporates accelerations and decelerations in a consistent and reproducible fashion.

Key words: aerobic capacity, acceleration, deceleration, endurance, HR, running speed.

\section{Introduction}

Team sports such as soccer, basketball and volleyball are characterized by frequent changes in activity patterns, mostly consisting of repetitive high-intensity actions (e.g. sprints,

\footnotetext{
1 - School of Physical Education and Sports Science, University of Thessaly, Karies, Trikala, 42100, Greece.

2 - School of Physical Education and Sports Science, Democritus University of Thrace, Komotini, 69100, Greece.

3 - Department of Sports Science and Clinical Biomechanics, SDU Sport and Health Sciences Cluster (SHSC), University of Southern Denmark, Odense, Denmark.

4 - Sport and Health Sciences, College of Life and Environmental Sciences, University of Exeter, Exeter, United Kingdom.

5 - Center of Health Science, Faculty of Health Sciences, University of the Faroe Islands, Jónas Broncksgøta 25, 3rd floor, Tórshavn, Faroe Islands.

6 - Center of Health and Human Performance, Department of Food and Nutrition, and Sports Science, University of Gothenburg, Gothenburg, Sweden.
} 
decelerations, accelerations, jumping, sliding, shooting and shuffling) interrupted by periods of low-to-moderate intensity or even standing still (McInnes et al., 1995; Mikołajec, 2012, 2017; Mroczek et al., 2014; Puente et al., 2017). Cardiovascular loading and muscle and blood responses during basketball and soccer games, indicate mobilization of all energy systems, i.e. oxidative phosphorylation, the ATPphosphocreatine (CP) system and glycolysis, with anaerobic loading being highly important during the most intense periods of play (Krustrup et al., 2006; McInnes et al., 1995; Mohr et al., 2016). In team sports, endurance refers to the ability to continuously carry out moderate-to-high-intensity activities, and performance is strongly associated with oxidative capacity, glycolytic rate, removal of lactate and other metabolic by-products, along with regulation of ion homeostasis (Bangsbo et al., 1995). Correlations between markers of these physiological systems and different match performance variables in a soccer game have been reported previously (Mohr et al., 2016). Improved sport-specific endurance is associated with more efficient match performance since players can cover greater distances and perform more highintensity actions in a game (Helgerud et al., 2001).

The amount of high-speed running is a crucial factor in soccer and distinguishes between distinct levels of competency (Mohr et al., 2003; Krustrup et al., 2015). Fatigue is often expressed as a decline in high-intensity running over time and is associated with high blood and muscle lactate levels, impaired buffering capacity, perturbations in ion balance and glycogen depletion (Mohr et al., 2015 , 2016). Soccer, basketball and volleyball are all intermittent-type sports characterized by a high rate of high-intensity actions creating a considerable metabolic and physiological overload, but they differ in terms of duration, muscle recruitment patterns, regulations and pitch/court dimensions (Chatzinikolaou et al., 2014; Draganidis et al., 2015; Narazaki, 2009).

Although numerous tests have been developed to assess the conditioning level of team sport athletes, most of them are continuous and their relevance to intermittent team sports has been questioned (Krustrup et al., 2003, 2005). The Yo-Yo Intermittent Endurance test level 2 (IE2) has been extensively utilized in the literature to monitor the sport-specific intermittent endurance capacity level and training adaptations in soccer players and other athletes in intermittent-type sports such as soccer, basketball, and volleyball (Bradley et al., 2011, 2014; Krustrup et al., 2015). The speed of the test progresses gradually, and the $2 \times 20-\mathrm{m}$ shuttle runs are followed by an active 5-s recovery phase. It has been stated recently that the Yo-Yo IE2 test scores have a positive correlation with running performance and $\mathrm{HR}$ responses in competitive elite male and female soccer players (Bradley et al., 2011, 2014) and that the training-induced alterations in soccer-specific endurance capacity of elite male and female players during a competitive season affect performance during the test (Bradley et al., 2014; Heisterberg et al., 2013). In a recent study examining physiological responses of the Yo-Yo IE2 test in untrained men and trained soccer players, it was observed that the Yo-Yo IE2 test was a sensitive tool to discriminate between performances at different fitness levels and also to impose alterations in muscle glycogen and $\mathrm{CP}$ content as well as in multiple metabolites such as blood lactate and glucose, plasma potassium, ammonia and free fatty acids (FFAs) (Krustrup et al., 2015). The intermittent nature of the Yo-Yo IE2 test makes it a valuable tool to evaluate endurance capacity of athletes participating in sports with an intermittent exercise pattern. The Yo-Yo IE2 is time- and cost-effective, easy to apply and frequently used by elite soccer teams to evaluate sport-specific endurance, but its validity and reproducibility have not been inadequately investigated even in soccer (Bradley et al., 2011). The Yo-Yo IE2 has been successfully utilized mostly in soccer, less often in basketball, and rarely in volleyball (Castagna et al., 2006). Despite basketball and volleyball being indoor team sports, they share common physiological elements, as the activity pattern of all these sports includes a variety of brief high intensity intermittent actions which tax the ATP-CP and glycolytic energy systems during practices and games and the oxidative phosphorylation system during the recovery phase (Sheppard et al., 2009). Hence, the Yo-Yo IE2 test could be utilized for the evaluation of sportspecific endurance capacity in outdoor and indoor team sports with an intermittent nature of activity patterns.

Although Yo-Yo IE2 performance has been shown to be correlated with maximal oxygen uptake $\left(\mathrm{VO}_{2 \mathrm{max}}\right)$, mostly in soccer players and, to a 
lesser extent, in basketball players, information for coaches and researchers regarding its reliability and reproducibility, especially in other team sports, is lacking (Bangsbo and Mohr, 2012). Therefore, the aim of this study was to (i) evaluate the reliability and reproducibility of both the physiological and overload features of the Yo-Yo IE2 in soccer, basketball and volleyball players; (ii) compare responses in overload variables of the test among testing trials in each sport discipline to determine whether a familiarization process is critical for this test; (iii) compare overall performance in this test among the three sport disciplines and its reproducibility across repeated trials; (iv) compare responses of overload variables of the test (i.e. distance covered, speed, acceleration, deceleration, heart rate, body load) across trials and between sport disciplines. We also hypothesized that heart rate, distance covered, speed, accelerations and decelerations would be reproducible in test-retest trials of the Yo-Yo IE2 in soccer, basketball and volleyball players and that there would be no differences between sport disciplines among sports in the responses of overload variables of the test.

\section{Methods}

\section{Participants and experimental overview}

A preliminary power analysis (a power of 0.9 , effect size $>0.55$, probability error of 0.05 ) based on previous similar studies on Yo-Yo testing validity and reliability (Bradley et al., 2011; Krustrup et al., 2003) indicated a sample size of 1820 total participants. Consequently, 29 competitive male soccer players, 15 competitive male basketball players and 14 competitive male volleyball players from local clubs were initially approached/interviewed, yet 20 soccer players (SP: age $21.9 \pm 1.5$ years; body mass $74.2 \pm 6.5 \mathrm{~kg}$; body height $1.77 \pm 0.06 \mathrm{~m}$; BMI $23.5 \pm 2.1 \mathrm{~kg} / \mathrm{m}^{2}$; body fat $16.6 \pm 4.4 \%$ ), 11 basketball players (BP: age $20.8 \pm$ 1.3 years; body mass $82.3 \pm 6.6 \mathrm{~kg}$; body height 1.86 $\pm 0.05 \mathrm{~m}$; BMI $23.7 \pm 1.2 \mathrm{~kg} / \mathrm{m}^{2}$; body fat $19.8 \pm 4.8 \%$ ) and 10 volleyball players (VP: age $20.9 \pm 1.1$ years; body mass $80.5 \pm 10.5 \mathrm{~kg}$; body height $1.86 \pm 0.08$ m; BMI $23.0 \pm 4.4 \mathrm{~kg} / \mathrm{m}^{2}$; body fat $18.6 \pm 4.4 \%$ ) finally volunteered to participate in this study. The inclusion criteria were: (1) participation at the competitive level for $\geq 6$ years ( $\geq 5$ practices/week); (2) no recent history ( $\leq 12$ months prior to the study) of muscle injury, illnesses/diseases; (3) no consumption of drugs, performance-enhancing supplements and medications. Written informed consent to participate in the study was provided by the participants after they had been informed about all risks, discomforts and benefits involved in the study. The procedures were in accordance with the 1975 Declaration of Helsinki, as revised in 2000, and approval was received from the institutional ethics committee (University of Thessaly).

A three-group, repeated-measures design was applied to evaluate the reliability of performance scores, physiological responses and overload characteristics to repeated (three trials) test-retest trials of the Yo-Yo IE2 tests. The study was performed one week following the completion of the participants' in-season period while they were still accustomed to training and match loads. During the week that preceded the experimental phase no training was performed by the participants. Players were measured at the same time of day (anthropometric measurements: 09.0011.00 a.m.; Yo-Yo IE2: 6.00-8.00 p.m.) and under the same environmental conditions. Two familiarization sessions of the Yo-Yo IE2 were allowed before the study. Yo-Yo IE2 testing was performed on a soccer pitch for SP and on an outdoor court for BP and VP, three times with a 7day recovery period between testing trials. During testing, participants' performance was monitored and their HR and overload characteristics (speed, accelerations, decelerations, distance, body load) were recorded.

\section{Measurements}

Body mass was measured to the nearest 0.1 kg (Seca alpha 770, Vogel \& Halke Hamburg, Germany) and body height to the nearest $1 \mathrm{~mm}$ (Seca bodymeter 208, Vogel \& Halke Hamburg, Germany). Body composition was assessed using dual-energy X-ray absorptiometry (DXA, Lunar DPX PRO) as described (Vlachopoulos et al., 2017).

The Yo-Yo IE2 was performed as previously described (Bradley et al., 2011). The HR was measured in 5-s intervals during the tests and data were subsequently analyzed using Polar Team2software (Polar team 2, Polar, Kempele, Finland). During testing, a high-time-resolution GPS device (15 Hz; GPSport, Canberra, Australia) was used to measure the distance covered, speed (mean and maximum), accelerations (mean values of covered distances at $0-2 \mathrm{~m} / \mathrm{s}^{2}, 2-3 \mathrm{~m} / \mathrm{s}^{2}$ and 
$>3 \mathrm{~m} / \mathrm{s}^{2}$ ), decelerations (mean values of covered distances at $0-2 \mathrm{~m} / \mathrm{s}^{2}, 2-3 \mathrm{~m} / \mathrm{s}^{2}$ and $\left.>3 \mathrm{~m} / \mathrm{s}^{2}\right)$, and body load (an arbitrary measure of total external mechanical stress using accelerometry data) (Cummins and Orr, 2015).

Statistical Analyses

One-way repeated measures ANOVA was used to detect differences in Yo-Yo IE2 performance, HR and overload responses. When a significant interaction was detected, data were then analyzed using a Bonferroni post-hoc test. The intra-class correlation coefficient index (ICC) and coefficients of variation $(\mathrm{CV})$ were used to analyze the reliability of the test. The magnitude for correlation coefficients was considered trivial $(\mathrm{r}<$ $0.1)$, small $(\mathrm{r}=0.1-0.3)$, moderate $(\mathrm{r}=0.3-0.5)$, large $(r=0.5-0.7)$, very large $(r=0.7-0.9)$, nearly perfect $(r>0.9)$ or perfect $(r=1.0)$ (Hopkins et al., 2009). Confidence intervals (CI) were also calculated for all ICCs. Statistical significance was set at $p<0.05$. Statistical tests were processed using SPSS software (SPSS Statistics for Windows, version 22, Chicago, IL, USA).

\section{Results}

Results are presented as means $\pm \mathrm{SD}$. While total body fat and was comparable between groups, SP had lower $(p<0.05)$ body mass and body height than BP and VP.

Figures 1 and 2 compare the total distance, heart rate, body load, speed, acceleration and deceleration responses during the Yo-Yo IE2 among trials and sport disciplines. In BP and VP, but not in SP, Yo-Yo IE2 performance increased ( $p$ $<0.05)$ during the second and third trials compared to the first one (Figure 1A). Yo-Yo IE2 performance of SP was greater $(p<0.05)$ compared to VP and BP in all trials. Performance of BP was greater $(p<$ 0.05 ) than that of VP in trials 2 and 3, but not in trial 1 (Figure 1A). Mean HR (Figure 1B) in the total sample, as well as in individual groups, was similar in trials 1 and 2 , but decreased $(p<0.05)$ in trial 3 (Figure 2A). No differences in mean HR response were detected between groups. Maximal HR (Figure 1C) and body load (Figure 1F) were similar between trials and groups during the test. Mean speed(Figure 1D) was similar among trials and groups, with the exception of BP which exhibited a greater value in trial 3 than in trials $1(p$ $<0.05)$ and $2(p<0.05)$. Maximum speed (Figure 1E) was similar among trials and groups, except for BP who exhibited a greater value in trial 1 than in trials $2(p<0.05)$ and $3(p<0.05)$. Mean distance covered with accelerations of $<2 \mathrm{~m} / \mathrm{s}^{2}$ (Figure 2A) was similar between groups in all trials. When the three Yo-Yo IE2 trials were compared, BP only demonstrated lower values in trial 1 compared to trials $2(p<0.05)$ and $3(p<0.05)$. Distance covered with accelerations at $2-3 \mathrm{~m} / \mathrm{s}^{2}$ (Figure 2B) was smaller in VP than in SP in all testing trials $(p<$ 0.05 ). Distance covered with accelerations of $>3$ $\mathrm{m} / \mathrm{s}^{2}$ (Figure $2 \mathrm{C}$ ) was similar between groups in all trials, except for the lower value in VP compared to SP in trial $3(p<0.05)$. No differences were noted among trials for all groups for distance covered with accelerations at $2-3 \mathrm{~m} / \mathrm{s}^{2}$ and $>3 \mathrm{~m} / \mathrm{s}^{2}$. Distances covered with decelerations of $<2 \mathrm{~m} / \mathrm{s}^{2}$ and $2-3 \mathrm{~m} / \mathrm{s}^{2}$ (Figures 2D-E) were similar between groups in all trials. However, distance covered with decelerations of $>3 \mathrm{~m} / \mathrm{s}^{2}$ (Figure $2 \mathrm{~F}$ ) was lower $(p<0.05)$ in VP and BP than in SP in all trials. No differences were noted between trials for all groups for all deceleration zones.

Table 1 provides the reliability coefficients (intra-class correlation coefficients and confidence intervals) for all variables tested. In SP, ICCs ranged from small to nearly perfect, i.e. 0.870 for total distance, 0.181 for average velocity, 0.519 for maximum velocity, 0.805 for mean HR, 0.848 for maximum HR and 0.888 for body load. In BP, ICCs ranged from small to nearly perfect, i.e. 0.767 for total distance, 0.127 for average velocity, 0.767 for maximum velocity, 0.931 for mean $H R, 0.933$ for maximum HR and 0.631 for body load. In VP, ICCs ranged from large to nearly perfect, i.e. 0.965 for total distance, 0.779 for average velocity, 0.790 for maximum velocity, 0.751 for mean $\mathrm{HR}, 0.593$ for maximum HR and 0.966 for body load. The CVs for distance, mean speed, HR responses, and acceleration and deceleration scores for test-retest trials ranged from 1.2 to $12.5 \%$, with no differences observed between different sport disciplines. 
A

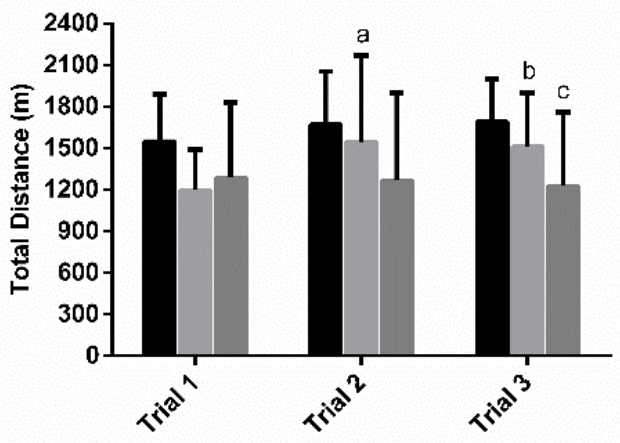

B

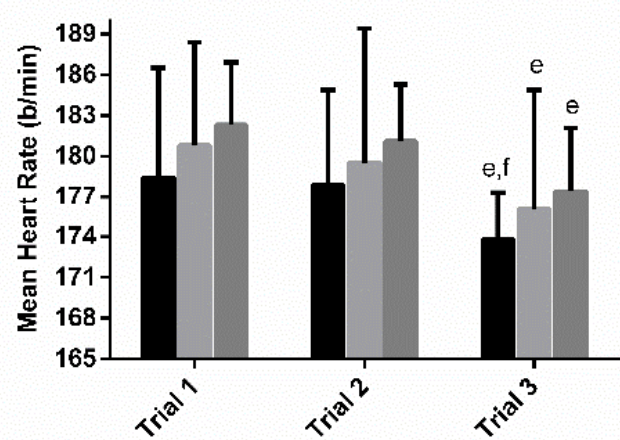

C

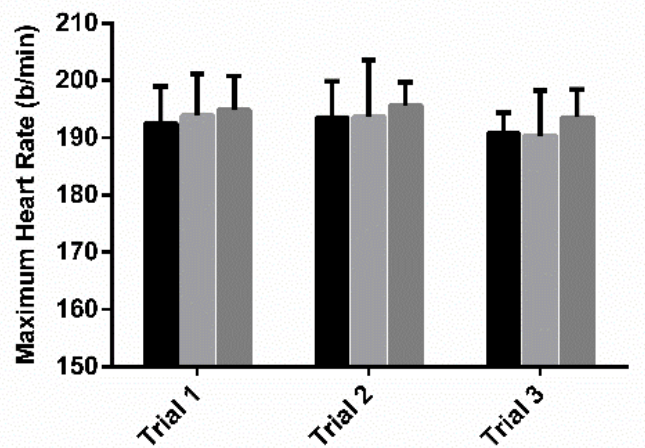

D

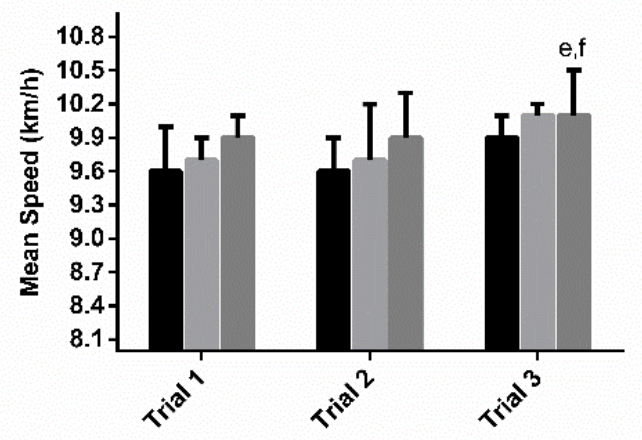

E

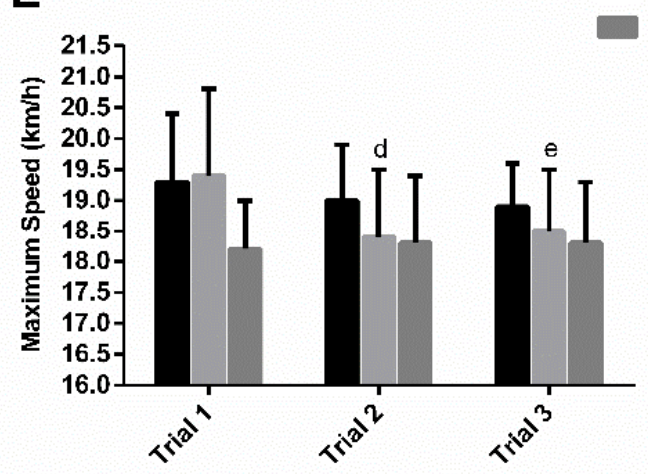

$\mathbf{F}$

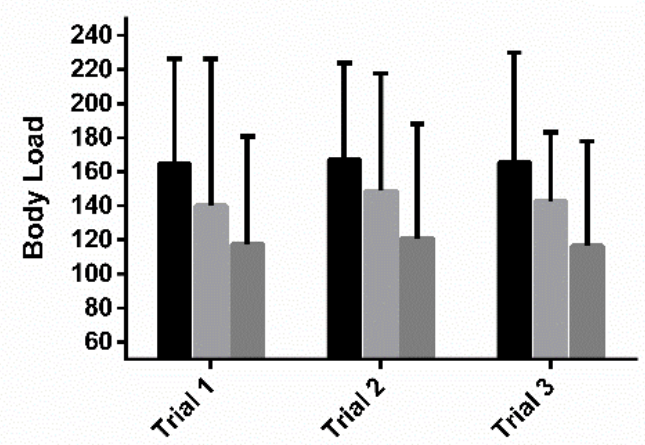

Figure 1

Total distance covered $(A)$, mean $(B)$ and maximum $(C) H R$ responses, mean $(D)$ and maximum (E) speed recorded and body load (F) measured during the Yo-Yo IE2 test. $S P$, soccer players; $B P$, basketball players; VP, volleyball players; ' ${ }^{a}$ significant difference

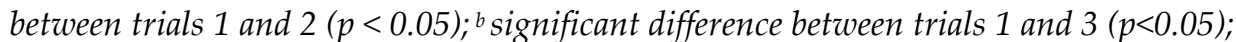
${ }^{e}$ significant difference between SP and VP in trial $3(p<0.05)$. 
A

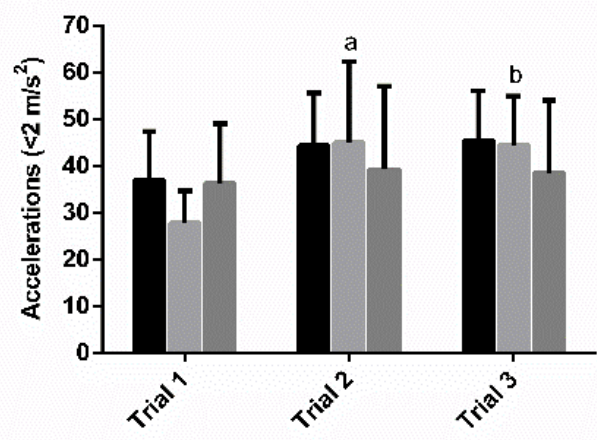

B

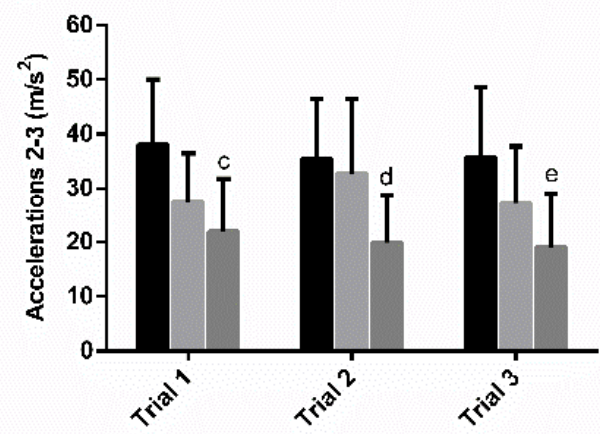

C

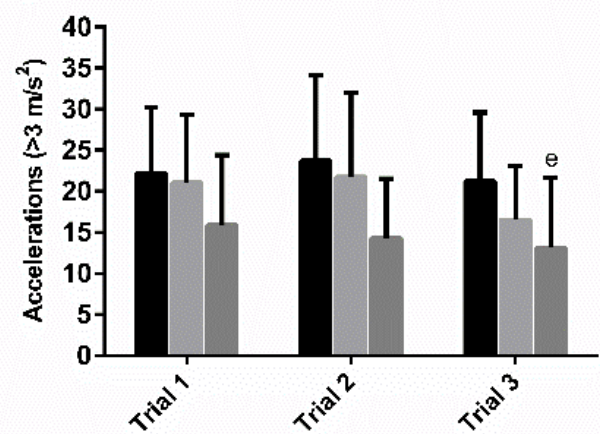

D
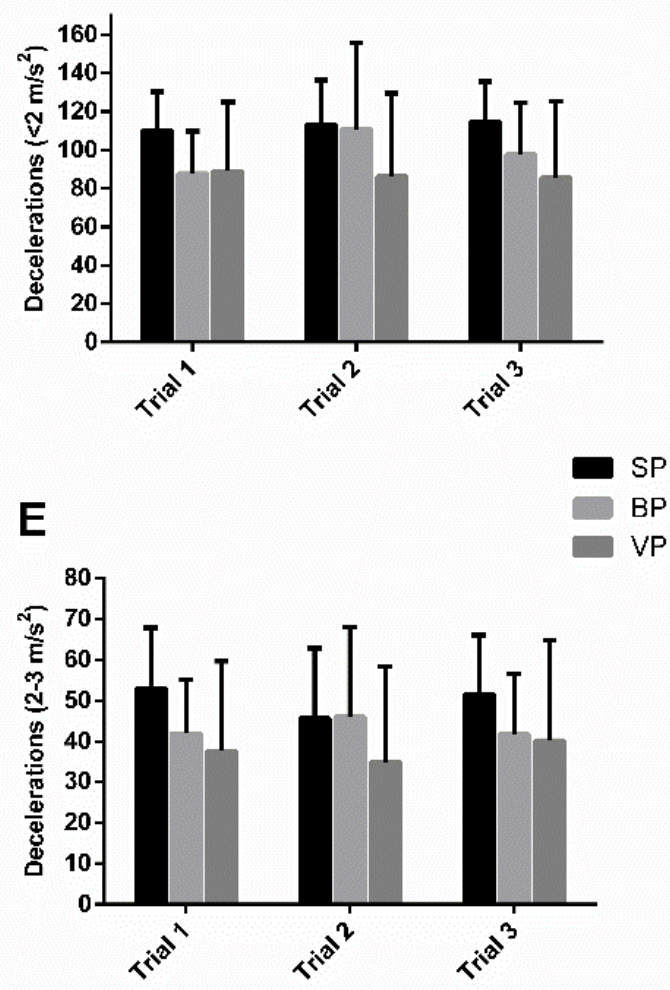

$\mathbf{F}$

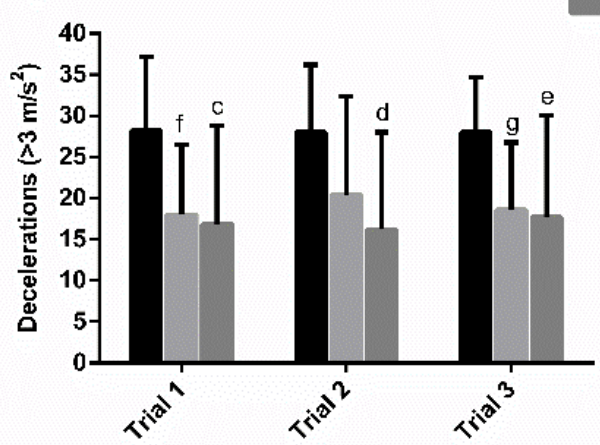

Figure 2

Mean distance covered with accelerations of $<2 \mathrm{~m} / \mathrm{s}^{2}(A), 2-3 \mathrm{~m} / \mathrm{s}^{2}(B)$, and $>3 \mathrm{~m} / \mathrm{s}^{2}(C)$ and decelerations of $<2 \mathrm{~m} / \mathrm{s}^{2}(D), 2-3 \mathrm{~m} / \mathrm{s}^{2}(E)$, and $>3 \mathrm{~m} / \mathrm{s}^{2}(F)$ during the $Y_{0}-Y_{O}$ IE2 test. SP, soccer players; $B P$, basketball players; $V P$, volleyball players; ${ }^{a}$ significant difference between trials 1 and 2 in $B P(p<0.05)$; ${ }^{b}$ significant difference between trials 1 and 3 in $B P(p<0.05)$; significant difference between SP and VP in trial $1(p<0.05)$; ${ }^{a}$ significant difference between SP and VP in trial $2(p<0.05)$; ${ }^{e}$ significant difference between SP and VP in trial $3(p<0.05)$. 
Table 1

Intra-class correlation coefficients and confidence intervals for all variables tested.

\begin{tabular}{|c|c|c|c|c|c|c|c|c|c|}
\hline \multirow[b]{3}{*}{ Variables } & \multicolumn{3}{|c|}{ SP } & \multicolumn{3}{|c|}{ BP } & \multicolumn{3}{|c|}{ VP } \\
\hline & \multirow[t]{2}{*}{ ICC } & \multicolumn{2}{|c|}{$95 \%$ CI } & \multirow[t]{2}{*}{ ICC } & \multicolumn{2}{|c|}{$95 \% \mathrm{CI}$} & \multirow[t]{2}{*}{ ICC } & \multicolumn{2}{|c|}{$95 \% \mathrm{CI}$} \\
\hline & & LB & UB & & LB & UB & & LB & UB \\
\hline Total Distance & 0.870 & 0.691 & 0.953 & 0.767 & 0.319 & 0.937 & 0.965 & 0.898 & 0.991 \\
\hline Mean Speed & 0.181 & -0.945 & 0.702 & 0.127 & -1.556 & 0.764 & 0.779 & 0.353 & 0.940 \\
\hline Maximum Speed & 0.519 & -0.141 & 0.825 & 0.767 & 0.317 & 0.937 & 0.790 & 0.384 & 0.943 \\
\hline Average HR & 0.805 & 0.521 & 0.932 & 0.931 & 0.799 & 0.981 & 0.751 & 0.270 & 0.933 \\
\hline Maximum HR & 0.848 & 0.640 & 0.945 & 0.933 & 0.804 & 0.982 & 0.593 & -1.191 & 0.890 \\
\hline Body Load & 0.888 & 0.734 & 0.959 & 0.631 & -0.082 & 0.900 & 0.966 & 0.902 & 0.991 \\
\hline $\operatorname{Acc}\left(0-2 \mathrm{~m} / \mathrm{s}^{2}\right)$ & 0.251 & -0.778 & 0.728 & -0.192 & -2.490 & 0.678 & 0.887 & 0.669 & 0.970 \\
\hline $\operatorname{Acc}\left(2-3 \mathrm{~m} / \mathrm{s}^{2}\right)$ & 0.454 & -0.298 & 0.801 & 0.845 & 0.546 & 0.958 & 0.806 & 0.433 & 0.948 \\
\hline $\operatorname{Acc}\left(>3 \mathrm{~m} / \mathrm{s}^{2}\right)$ & 0.774 & 0.464 & 0.918 & 0.523 & -0.398 & 0.871 & 0.656 & -0.009 & 0.907 \\
\hline $\operatorname{Dec}\left(0-2 \mathrm{~m} / \mathrm{s}^{2}\right)$ & 0.772 & 0.459 & 0.917 & 0.623 & -0.105 & 0.898 & 0.918 & 0.759 & 0.978 \\
\hline $\operatorname{Dec}\left(2-3 \mathrm{~m} / \mathrm{s}^{2}\right)$ & 0.799 & 0.523 & 0.927 & 0.879 & 0.646 & 0.967 & 0.900 & 0.706 & 0.973 \\
\hline $\operatorname{Dec}\left(>3 \mathrm{~m} / \mathrm{s}^{2}\right)$ & 0.780 & 0.478 & 0.920 & 0.870 & 0.619 & 0.965 & 0.899 & 0.703 & 0.973 \\
\hline $\begin{array}{r}\text { SP, soccer pla } \\
\text { Coefficient; CI, C }\end{array}$ & ; $B P$ & sketbal & layers & $P$, voll & ball plo & $\begin{array}{l}\text { rs; ICC } \\
\text { r Bour }\end{array}$ & ntracl & $\begin{array}{l}\text { Corre } \\
\text { elerati }\end{array}$ & ; Dec, \\
\hline
\end{tabular}

\section{Discussion}

The principal finding in the present investigation suggests that the Yo-Yo IE2 test is a reliable protocol for evaluating intermittent endurance capacity in both indoor and outdoor sports, i.e. soccer, basketball and volleyball. It appears that HR and overload characteristics, 
except for mean speed, are efficiently reproduced in repeated testing trials irrespective of the type of sport. Previous studies have evaluated the testretest reproducibility of the Yo-Yo IE2 test in elite female and male soccer players (Bradley et al., 2012; Krustrup et al., 2003), but such data is lacking for basketball and volleyball players (Bradley et al., 2011, 2012). This is the first study to examine the reliability and reproducibility of physiological and overload responses of the Yo-Yo IE2 test in competitive volleyball, basketball and soccer players. We revealed that Yo-Yo IE2 performance of BP and VP was comparable when this test was repeated just one week apart, demonstrating a very low coefficient of variation (CV of $4.5 \%$ ), which is similar to that reported for elite male soccer players (3.9\%) (Bradley et al., 2011) and the Yo-Yo IR1 test (5\%) (Krustrup et al., 2003), but lower than that reported for the Yo-Yo IR2 test (8-10\%) (Bangsbo et al., 2008; Krustrup et al., 2006). These low CV values are close to those reported for laboratory tests assessing $\mathrm{VO} 2 \mathrm{max}$ and lactate threshold variables (Grant et al., 2002; Pfitzinger and Freedson, 1998).

Yo-Yo IE2 performance was slightly lower compared to that reported by others for SP (Povoas et al., 2016), probably due to differences in participants' levels of intermittent endurance capacity (Bradley et al., 2014). In general, SP performed better than VP and BP in this study, which agrees with the existing standards published for these sports (Rampinini et al., 2010). Overall, the reliability of total distance covered in repeated Yo-Yo IE2 trials was almost perfect for all groups, suggesting a high degree of reproducibility irrespective of the sport. In contrast, the reliability for mean speed was still large, but lower than the other performance variables for all groups. This may be attributed to a different running technique in players of different sports, which results in a versatile acceleration and deceleration running pattern. Considering the observed slightly better performance from test to test, the players became more familiar with the test and they developed a specific adaptation to the test, resulting in potentially better running economy (Folland et al., 2017). Interestingly, performance was progressively improved from test to test, indicating that multiple familiarization trials are needed for the test to reflect the actual test performance.

Maximum HR responses appear to be quite reliable and in accordance with other studies (Bangsbo et al., 2008; Bradley et al., 2011; Krustrup et al., 2015). The average HR responses observed in the study followed a downturn from the first to the third trial suggesting that the players acquired an adaptation to the test, probably as a result of better technique and control of accelerations, decelerations and turns during the test. This is also evident by the fact that all athletes exhibited high reliability scores for accelerations and decelerations, especially in those $>2 \mathrm{~m} / \mathrm{s} 2$. HR response demonstrated a progressive rise during the Yo-Yo IE2 test, suggesting a progressive rise in oxygen uptake (Bangsbo et al., 2008). This rise reached age-predicted maximal levels and was reproducible from trial to trial in all athletic groups. Although further validation of submaximal and maximal HR responses recorded during the Yo-Yo IE2 is needed, especially in BP and $\mathrm{VP}$, it appears that athletes can reach maximal HR values with consistency in multiple trials performed one week apart.

ICCs and CVs for the Yo-Yo IE2 were similar to those reported previously for SP on the Yo-Yo IR1 test (Krustrup et al., 2003) in terms of physiological and performance responses. However, mean speed ICC values were lower compared to those obtained for other performance measures, suggesting that competitive athletes, especially SP and BP, may have difficulty maintaining the same speed level across test stages. This issue may be resolved by introducing more familiarization trials. Interestingly, VP demonstrated higher ICC values for mean speed compared to SP and BP.

For the first time, it has been shown that the Yo-Yo IE2 incorporates a considerable amount of low-, moderate-, and high-intensity accelerations and decelerations, which are considered of paramount importance for success in soccer, basketball and volleyball. In fact, all athletes demonstrated large ICCs and low CVs for accelerations and decelerations, suggesting consistent reproducibility of these elements during testing. Although there is a small number of studies suggesting that the Yo-Yo IE may be a valid and reliable test for BP (Padulo et al., 2016), no data exist for VP. It seems that GPS analysis of the activity data of this test further corroborates that it 
may be used for BP and VP as it is already for SP.

The Yo-Yo IE2 consistently confirmed a higher level of intermittent endurance capacity of SP compared to BP and VP and that of BP compared to VP, which has also been described in the literature, further corroborating the capacity of this test to consistently discriminate between different levels of intermittent endurance capacity (Deprez et al., 2015).

In contrast to laboratory testing, the use of the Yo-Yo IE2 test enables practitioners to assess the intermittent endurance capacity of all players in a team more frequently, with minimal equipment, at low cost and in a time-efficient manner (Stolen et al., 2005; Svensson and Drust, 2005). Moreover, it appears that the test is capable of quantifying the intermittent endurance capacity of players of different team sports (outdoors and indoors), such as soccer, basketball and volleyball, which share some common elements of the physiological overload. Accordingly, basketball and volleyball practitioners could acquire information for monitoring training adaptations and planning the training micro- and macro-cycle. In addition, the Yo-Yo IE2 test could be also a time-effective option of intermittent endurance exercise for coaches which can efficiently replace an endurance training session, given the fact that the test can highly tax all energy systems and impose multiple physiological responses (Krustrup et al., 2015). Furthermore, the Yo-Yo IE2 test is reproducible not only in soccer, but also in basketball and volleyball players and thus may be a sensitive tool to differentiate between different levels of intermittent endurance capacity. However, it is essential for players to perform familiarization sessions prior to actual testing to obtain a concrete estimation of their performance level.

\section{Practical Implications}

The Yo-Yo IE2 appears to be a reliable alternative for evaluating intermittent endurance capacity among different codes of team sports. This test incorporates accelerations and decelerations in a consistent and reproducible fashion. Players should be adequately familiarized prior to actual testing to ensure valid assessment of intermittent endurance capacity.

\section{Acknowledgements}

Authors wish to thank all participants for their commitment to the study. The study was supported by departmental funding.

\section{References}

Bangsbo J, Aagaard T, Olsen M, Kiens B, Turcotte LP, Richter EA. Lactate and $\mathrm{H}^{+}$uptake in inactive muscles during intense exercise in man. J Physiol, 1995; 488: 219-229

Bangsbo J, Iaia FM, Krustrup P. The Yo-Yo intermittent recovery test : a useful tool for evaluation of physical performance in intermittent sports. Sports Med, 2008; 38: 37-51

Bangsbo J, Mohr M. Fitness testing in football. Espergaerde: Bangsbosport; 2012

Bradley PS, Bendiksen M, Dellal A, Mohr M, Wilkie A, Datson N, Orntoft C, Zebis M, Gomez-Diaz A, Bangsbo J, Krustrup P. The application of the Yo-Yo intermittent endurance level 2 test to elite female soccer populations. Scand J Med Sci Sports, 2014; 24: 43-54

Bradley PS, Di Mascio M, Bangsbo J, Krustrup P. The maximal and sub-maximal versions of the Yo-Yo intermittent endurance test level 2 are simply reproducible, sensitive and valid. Eur J Appl Physiol, 2012; 112: 1973-1975

Bradley PS, Mohr M, Bendiksen M, Randers MB, Flindt M, Barnes C, Hood P, Gomez A, Andersen JL, Di Mascio M, Bangsbo J, Krustrup P. Sub-maximal and maximal Yo-Yo intermittent endurance test level 2: HR response, reproducibility and application to elite soccer. Eur J Appl Physiol, 2011; 111: 969-978

Castagna C, Impellizzeri FM, Belardinelli R, Abt G, Coutts A, Chamari K, D'Ottavio S. Cardiorespiratory responses to Yo-yo Intermittent Endurance Test in nonelite youth soccer players. J Strength Cond Res, 20: 2006; 326-330

Chatzinikolaou A, Draganidis D, Avloniti A, Karipidis A, Jamurtas AZ, Skevaki CL, Tsoukas D, Sovatzidis 
A, Theodorou A, Kambas A, Papassotiriou I, Taxildaris K, Fatouros I. The microcycle of inflammation and performance changes after a basketball match. J Sports Sci, 2014; 32: 870-882

Cummins C, Orr R. Analysis of Physical Collisions in Elite National Rugby League Match Play. Int J Sports Physiol Perform, 2015; 10: 732-739

Deprez D, Fransen J, Lenoir M, Philippaerts R, Vaeyens R. The Yo-Yo intermittent recovery test level 1 is reliable in young high-level soccer players. Biol Sport, 2015; 32: 65-70

Draganidis D, Chatzinikolaou A, Avloniti A, Barbero-Alvarez JC, Mohr M, Malliou P, Gourgoulis V, Deli CK, Douroudos, II, Margonis K, Gioftsidou A, Flouris AD, Jamurtas AZ, Koutedakis Y, Fatouros IG. Recovery kinetics of knee flexor and extensor strength after a football match. PLoS One, 2015; 10: e0128072

Folland JP, Allen SJ, Black MI, Handsaker JC, Forrester SE. Running Technique is an Important Component of Running Economy and Performance. Med Sci Sports Exerc, 2017; 49: 1412-1423

Grant S, McMillan K, Newell J, Wood L, Keatley S, Simpson D, Leslie K, Fairlie-Clark S. Reproducibility of the blood lactate threshold, 4 mmol. $\mathrm{l}^{(-1)}$ marker, HR and ratings of perceived exertion during incremental treadmill exercise in humans. Eur J Appl Physiol, 2002; 87: 159-166

Heisterberg MF, Fahrenkrug J, Krustrup P, Storskov A, Kjaer M, Andersen JL. Extensive monitoring through multiple blood samples in professional soccer players. J Strength Cond Res, 2013; 27(5): 1260-1271

Helgerud J, Engen LC, Wisloff U, Hoff J. Aerobic endurance training improves soccer performance. Med Sci Sports Exerc, 2001; 33: 1925-1931

Hopkins WG, Marshall SW, Batterham AM, Hanin J. Progressive statistics for studies in sports medicine and exercise science. Med Sci Sports Exerc, 2009; 41: 3-13

Krustrup P, Ermidis G, Mohr M. Sodium bicarbonate intake improves high-intensity intermittent exercise performance in trained young men. J Int Soc Sports Nutr, 2015; 12: 25

Krustrup P, Mohr M, Amstrup T, Rysgaard T, Johansen J, Steensberg A, Pedersen PK, Bangsbo J. The yo-yo intermittent recovery test: physiological response, reliability, and validity. Med Sci Sports Exerc, 2003; 35: 697-705

Krustrup P, Mohr M, Ellingsgaard H, Bangsbo J. Physical demands during an elite female soccer game: importance of training status. Med Sci Sports Exerc, 2005; 37: 1242-1248

Krustrup P, Mohr M, Nybo L, Jensen JM, Nielsen JJ, Bangsbo J. The Yo-Yo IR2 test: physiological response, reliability, and application to elite soccer. Med Sci Sports Exerc, 2006; 38: 1666-1673

McInnes SE, Carlson JS, Jones CJ, McKenna MJ. The physiological load imposed on basketball players during competition. J Sports Sci, 1995; 13: 387-397

Mikolajec K, Waskiewicz Z, Maszczyk A, Bacik B, Kurek P, Zając, A. Effects of stretching and strength exercises on speed and power abilities in male basketball players. Isokinet Exerc Sci. 2012; 20(1): 61-69

Mikołajec K, Maszczyk A, Chalimoniuk M, Langfort J. Gołaś A, Zajc A. The influence of strength exercises of the lower limbs on postural stability: A possible role of the autonomic nervous system. Isokinet Exerc Sci, 2017; 25(2): 79-89

Mohr M, Draganidis D, Chatzinikolaou A, Barbero-Alvarez JC, Castagna C, Douroudos I, Avloniti A, Margeli A, Papassotiriou I, Flouris AD, Jamurtas AZ, Krustrup P, Fatouros IG. Muscle damage, inflammatory, immune and performance responses to three football games in 1 week in competitive male players. Eur J Appl Physiol, 2016; 116: 179-193

Mohr M, Krustrup P, Bangsbo J. Fatigue in soccer: a brief review. J Sports Sci, 2005; 23: 593-599

Mroczek D, Januszkiewicz A, Kawczynski AS, Borysiuk Z, Chmura J. Analysis of male volleyball players' motor activities during a top level match. J Strength Cond Res, 2014; 28: 2297-2305

Narazaki K, Berg K, Stergiou N, Chen B. Physiological demands of competitive basketball. Scand J Med Sci Sports 2009; 19: 425-432 
Padulo J, Bragazzi NL, Nikolaidis PT, Dello Iacono A, Attene G, Pizzolato F, Dal Pupo J, Zagatto AM, Oggianu M, Migliaccio GM. Repeated Sprint Ability in Young Basketball Players: Multi-direction vs. OneChange of Direction (Part 1). Front Physiol, 2016; 7: 133

Pfitzinger P, Freedson PS. The reliability of lactate measurements during exercise. Int J Sports Med, 1998; 19: 349-357

Povoas SC, Castagna C, Soares JM, Silva PM, Lopes MV, Krustrup P. Reliability and validity of Yo-Yo tests in 9- to 16-year-old football players and matched non-sports active schoolboys. Eur J Sport Sci, 2016; 16: 755-763

Puente C, Abian-Vicen J, Areces F, Lopez R, Del Coso J. Physical and Physiological Demands of Experienced Male Basketball Players During a Competitive Game. J Strength Cond Res, 2017; 31: 956-962

Rampinini E, Sassi A, Azzalin A, Castagna C, Menaspa P, Carlomagno D, Impellizzeri FM. Physiological determinants of Yo-Yo intermittent recovery tests in male soccer players. Eur J Appl Physiol, 2010; 108: 401-409

Sheppard JM, Chapman DW, Gough C, McGuigan MR, Newton RU. Twelve-month training-induced changes in elite international volleyball players. J Strength Cond Res, 2009; 23: 2096-2101

Stolen T, Chamari K, Castagna C, Wisloff U. Physiology of soccer: an update. Sports Med, 2005; 35(6): 501-536

Svensson M, Drust B. Testing soccer players. J Sports Sci, 2005; 23(6): 601-618

Vlachopoulos D, Barker AR, Williams CA, SA AR, Knapp KM, Metcalf BS, Fatouros IG, Moreno LA, GraciaMarco L. The Impact of Sport Participation on Bone Mass and Geometry in Male Adolescents. Med Sci Sports Exerc, 2017; 49: 317-326

\section{Corresponding author:}

\section{Ioannis G. Fatouros, Ph.D}

School of Physical Education and Sports Sciences,

University Campus, Trikala, Karies, 42100, Greece,

Tel +30-24310-47055,

E-mail: ifatouros@pe.uth 